\title{
Estimate of the Basic Reproduction Number for COVID-19: A Systematic Review and Meta-analysis
}

\author{
Yousef Alimohamadi ${ }^{1,2}$, Maryam Taghdir ${ }^{3}$, Mojtaba Sepandi ${ }^{3,4}$ \\ 'Pars Advanced and Minimally Invasive Medical Manners Research Center, Pars Hospital, Iran University of Medical Sciences, Tehran, Iran; \\ ${ }^{2}$ Department of Epidemiology and Biostatistics, School of Public Health, Tehran University of Medical Sciences, Tehran, Iran; ${ }^{3}$ Health Research \\ Center, Lifestyle Institute, Baqiyatallah University of Medical Sciences, Tehran, Iran; ${ }^{4}$ Department of Epidemiology and Biostatistics, Faculty of \\ Health, Baqiyatallah University of Medical Sciences, Tehran, Iran
}

Objectives: The outbreak of coronavirus disease 2019 (COVID-19) is one of the main public health challenges currently facing the world. Because of its high transmissibility, COVID-19 has already caused extensive morbidity and mortality in many countries throughout the world. An accurate estimation of the basic reproduction number $\left(R_{0}\right)$ of COVID-19 would be beneficial for prevention programs. In light of discrepancies in original research on this issue, this systematic review and meta-analysis aimed to estimate the pooled $R_{0}$ for COVID-19 in the current outbreak.

Methods: International databases (including Google Scholar, Science Direct, PubMed, and Scopus) were searched to identify studies conducted regarding the $R_{0}$ of COVID-19. Articles were searched using the following keywords: "COVID-19" and "basic reproduction number" or " $R_{0}$." The heterogeneity among studies was assessed using the $I^{2}$ index, the Cochran $Q$ test, and $T^{2}$. A random-effects model was used to estimate $R_{0}$ in this study.

Results: The mean reported $R_{0}$ in the identified articles was $3.38 \pm 1.40$, with a range of 1.90 to 6.49 . According to the results of the random-effects model, the pooled $R_{0}$ for COVID-19 was estimated as 3.32 (95\% confidence interval, 2.81 to 3.82 ). According to the results of the meta-regression analysis, the type of model used to estimate $R_{0}$ did not have a significant effect on heterogeneity among studies ( $p=0.81$ ).

Conclusions: Considering the estimated $R_{0}$ for COVID-19, reducing the number of contacts within the population is a necessary step to control the epidemic. The estimated overall $R_{0}$ was higher than the World Health Organization estimate.

Key words: Basic reproduction number, COVID-19, Meta-analysis, Public health, Outbreak

\section{INTRODUCTION}

In December 2019, a series of pneumonia cases with no identified cause appeared in Wuhan, Hubei Province, China, with

Received: March 11, 2020 Accepted: March 20, 2020

Corresponding author: Mojtaba Sepandi, PhD

Health Research Center, Lifestyle Institute, Baqiyatallah University of

Medical Sciences, Tehran 143591-13189, Iran

E-mail:msepandi@gmail.com

This is an Open Access article distributed under the terms of the Creative Commons Attribution Non-Commercial License (https://creativecommons.org/licenses/by$\mathrm{nc} / 4.0 /$ ) which permits unrestricted non-commercial use, distribution, and reproduction in any medium, provided the original work is properly cited. clinical symptoms similar to viral pneumonia [1-3]. Most of the reported cases were in patients who worked or lived around the local Huanan Seafood Wholesale Market, where live animals were also sold [4]. This new virus infecting humans was initially named the 2019 novel coronavirus, and the World Health Organization (WHO) subsequently issued updated nomenclature, in which the virus is referred to as severe acute respiratory syndrome coronavirus 2 (SARS-CoV-2) and the disease that it causes is referred to as coronavirus disease 2019 (COVID-19) [5]. Because of its high contagiousness and morbidity, this infection is considered by WHO as a global emergency [6]. As a reflection of the high transmissibility of this viral infection, by Janu- 
ary 26, 2020 more than 2000 confirmed cases of COVID-19 had been identified in China, mainly in Wuhan [7]. This number then dramatically increased, with the number of confirmed cases in China reaching 66580 by February 15, 2020, with 1524 deaths [8]. Human-to-human transmission of COVID-19 was confirmed [9] and cases were reported in countries other than China [10]. Because of the high infectiousness of SARSCoV-2 among the susceptible population, the calculation of the basic reproduction number $\left(R_{0}\right)$ is essential for implementing prevention measures [1]. $R_{0}$ is an epidemiological metric that can be used to assess the contagiousness of infectious agents. This index presents the average number of new cases generated by an infected person $[11,12]$. Therefore, a high $R_{0}$ indicates that an infectious agent is highly contagious. Since the epidemic began in China, numerous papers have been published on this issue. However, because of discrepancies in the results of those studies, the current systematic review and meta-analysis aimed to estimate the pooled $R_{0}$ for the COVID-19 outbreak, using original articles published during 2020.

\section{METHODS}

\section{Search Strategy}

This systematic review and meta-analysis was performed to estimate the pooled $R_{0}$ of COVID-19 in articles published in international journals. International databases (including Google Scholar, Science Direct, PubMed, and Scopus) were searched to obtain studies conducted regarding the reproduction number of COVID-19. Articles were searched using the keywords "COVID-19" AND "basic reproduction number" OR " $R_{0}$ ".

\section{Study Selection and Data Extraction}

In the current study, all studies published in 2020 that estimated $R_{0}$ for COVID-19 were entered into the meta-analysis. The name of the first author, country, year of the study, model used to estimate $R_{0}$, and the estimated $R_{0}$ value (with a $95 \%$ confidence interval, $\mathrm{Cl}$ ) were extracted from the articles.

\section{Statistical Analysis}

Heterogeneity between studies was assessed using the $\mathrm{I}^{2}$ index, the Cochran $Q$ test, and $\mathrm{T}^{2}$. According to the $\mathrm{I}^{2}$ results, heterogeneity can classified into the following 3 categories: $\mathrm{I}^{2}<25 \%$ (low heterogeneity), $\mathrm{I}^{2}=25-75 \%$ (average heterogeneity), and $I^{2}>75 \%$ (high heterogeneity) [13]. Because of the high $I^{2}$ value that was calculated (99.4\%), as well as the signifi- cance of the Cochran $Q$ test $(p<0.001)$, a random-effects model was used to estimate $R_{0}$ in this study. The impact of covariates on the estimated $R_{0}$ was also assessed by univariate meta-regression. Data were analyzed using Stata version 11 (StataCorp., College Station, TX, USA).

\section{Ethics Statement}

As a systematic review, this study did not need ethical approval.

\section{RESULTS}

We identified 85 studies, of which 23 were duplicates, leaving 62 reports. A total of 55 reports passed the initial screening, and 23 reports passed the full-text assessment for eligibility (Figure 1). The reasons for exclusion were as follows: reporting of effective reproductive number instead of $R_{0}$ and insufficient data. Finally, we included 23 studies in this systematic review (Table 1). No studies were excluded due to poor quality. In the current study, 23 studies with 29 records that estimated the $R_{0}$

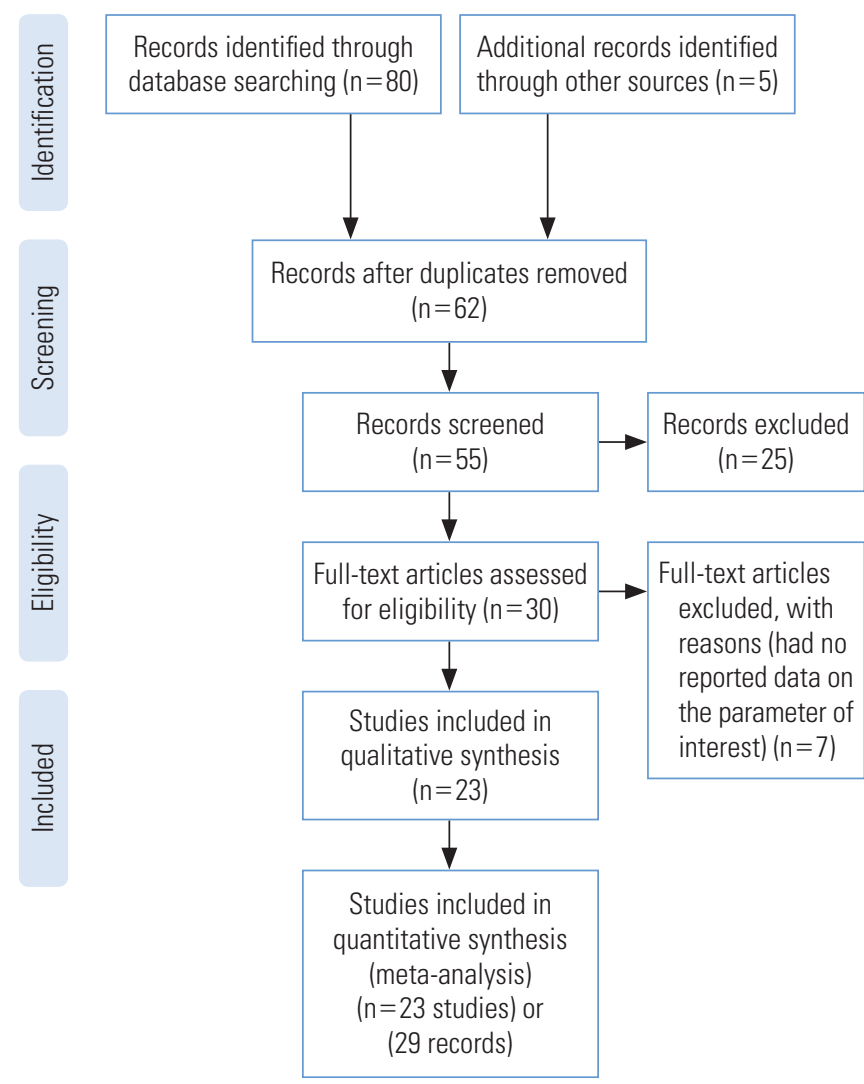

Figure 1. PRISMA (Preferred Reporting Items for Systematic Reviews and Meta-Analyses) flow diagram for the studies included in the current meta-analysis. 
Table 1. Descriptive characteristics of the studies included in the meta-analysis

\begin{tabular}{|c|c|c|c|c|c|}
\hline Study & Country & Model & No. of reproduction & LCL & UCL \\
\hline Wu et al., 2020 [14] & China & MCMC & 2.68 & 2.47 & 2.86 \\
\hline Shen et al., 2020 [15] & China & Dynamic compartmental model & 6.49 & 6.31 & 6.66 \\
\hline Liu et al., 2020 [16] & China & Statistical exponential growth model & 2.90 & 2.32 & 3.63 \\
\hline Liu et al., 2020 [16] & China & Statistical maximum likelihood estimation & 2.92 & 2.28 & 3.67 \\
\hline Read et al., 2020 [17] & China & Mathematical transmission model & 3.11 & 2.39 & 4.13 \\
\hline Majumder et al., 2020 [18] & China & IDEA & 2.55 & 2.00 & 3.10 \\
\hline Liu et al., $2020[11]$ & China & Mathematical model & 1.95 & 1.40 & 2.50 \\
\hline Zhao et al., 2020 [19] & China & Statistical exponential growth model & 2.24 & 1.96 & 2.55 \\
\hline Zhao et al., 2020 [19] & China & Statistical exponential growth model & 3.58 & 2.89 & 4.39 \\
\hline Imai et al., 2020 [20] & China & Mathematical model & 2.50 & 1.50 & 3.50 \\
\hline Riou et al., 2020 [21] & China & Stochastic simulations of early outbreak trajectories & 2.20 & 1.40 & 3.80 \\
\hline Tang et al., 2020 [22] & China & Mathematical SEIR-type epidemiological model & 6.47 & 5.71 & 7.23 \\
\hline Li et al., 2020 [23] & China & Statistical exponential growth model & 2.20 & 1.40 & 3.90 \\
\hline Zhang et al., 2020 [24] & China & Statistical maximum likelihood estimation & 2.28 & 2.06 & 2.52 \\
\hline Shen et al., 2020 [15] & China & Mathematical model & 4.71 & 4.50 & 4.92 \\
\hline Du et al., 2020 [25] & China & Statistical exponential growth model & 1.90 & 1.47 & 2.59 \\
\hline Muniz-Rodriguez et al., 2020 [26] & China & Statistical exponential growth model & 3.30 & 3.10 & 4.20 \\
\hline Zhou, 2020 [27] & China & SEIR model & 2.12 & 2.04 & 2.18 \\
\hline Liu et al., 2020 [28] & China & Statistical exponential growth model & 4.50 & 4.40 & 4.60 \\
\hline Liu et al., 2020 [28] & China & Statistical exponential growth model & 4.40 & 4.30 & 4.60 \\
\hline Li et al., 2020 [29] & China & Networked dynamic metapopulation model & 2.23 & 1.77 & 3.00 \\
\hline Park et al., 2020 [30] & China & MCMC & 3.10 & 2.10 & 5.70 \\
\hline Shao et al., 2020 [31] & China & Fudan-CCDC model & 3.32 & 3.25 & 3.40 \\
\hline Zhang et al., 2020 [32] & China & SEIO model & 5.50 & 5.30 & 5.80 \\
\hline Lai et al., 2020 [33] & China & Coalescent-based exponential growth and a birth-death skyline method & 2.60 & 2.10 & 5.10 \\
\hline Jung et al., 2020 [9] & China & MCMC & 2.10 & 2.00 & 2.20 \\
\hline Jung et al., 2020 [9] & China & MCMC & 3.20 & 2.70 & 3.70 \\
\hline Sanche et al., 2020 [34] & China & Statistical exponential growth model & 6.30 & 3.30 & 11.30 \\
\hline Sanche et al., 2020 [34] & China & Statistical exponential growth model & 4.70 & 2.80 & 7.60 \\
\hline
\end{tabular}

LCL, lower control limit; UCL, upper control limit; MCMC, Markov chain Monte Carlo; IDEA, incidence decay and exponential adjustment; SEIR, susceptible, exposed, infected, and resistant; CCDC, Chinese Center for Disease Control and Prevention; SEIQ, susceptible, exposed, infected and quarantined.

of COVID-19 were entered into the analysis. The studies used a broad range of methods to estimate $R_{0}$ for COVID-19. All the studies included in the meta-analysis were conducted in 2020 in China. The mean $R_{0}$ reported in the articles was calculated as $3.38 \pm 1.40$, with a range of 1.90 to 6.49 . More information is shown in Table 1.

\section{Pooled Estimation of Basic Reproduction Number}

According to the results of the random-effects model, the pooled $R_{0}$ for COVID-19 was estimated as $3.32(95 \% \mathrm{Cl}, 2.81$ to 3.82). This means that each person infected with COVID-19 transmitted the infection to between 3 and 4 susceptible peo- ple on average (Figure 2, Table 2). There was significant heterogeneity among studies $\left(I^{2}=99.4 \%, p\right.$ from the chi-square test for heterogeneity $<0.001$, and $\mathrm{T}^{2}=1.72$ ) (Table 2 ).

\section{Meta-regression}

The meta-regression analysis showed that the type of model used to estimate $R_{0}$ did not have a significant effect on heterogeneity among studies $(p=0.81)$. The distribution of the estimated $R_{0}$ according to the model used is shown in Figure 3 . The numbers on the $\chi$-axis in Figure 3 represent the type of method used to estimate $R_{0}$, using the following coding: stochastic Markov chain Monte Carlo method: 1, dynamic compartmental model; 2, statistical exponential growth model; 3, 


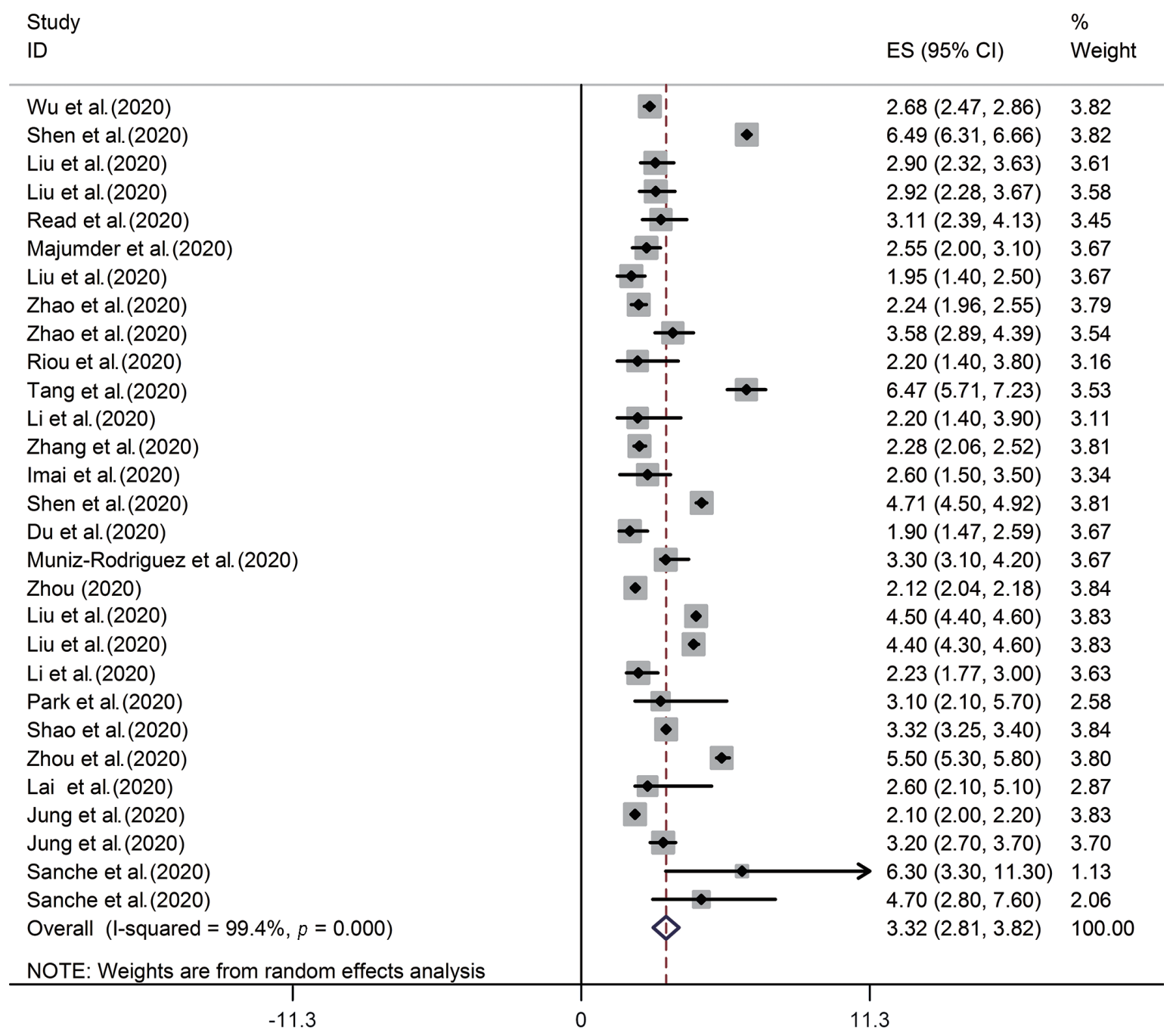

Figure 2. Forest plot of the estimated basic reproduction number of coronavirus disease 2019. ES, effect size; Cl, confidence interval.

Table 2. Pooled estimation of the basic reproduction number of coronavirus disease 2019

\begin{tabular}{lccc}
\hline Pooled estimate $(\mathbf{9 5 \%} \mathbf{C I})$ & $\mathbf{0}$ & $\mathbf{I}^{\mathbf{2}}$ & $\mathbf{T}^{\mathbf{2}}$ \\
\hline $3.32(2.81,3.82)$ & $<0.001$ & 99.4 & 1.72
\end{tabular}

$\mathrm{Cl}$, confidence interval.

statistical maximum likelihood estimation; 4, mathematical transmission model; 5, mathematical incidence decay and exponential adjustment; 6 , stochastic simulation of early outbreak trajectories; 7, mathematical susceptible, exposed, infected, and resistant (SEIR)-type epidemiological model; 8, other mathematical models; 9 , networked dynamics metapopulation model; 10, Fudan-Chinese Center for Disease Control and Prevention model; 11, susceptible, exposed, infected and quarantined (SEIQ) model; 12, coalescent-based exponen-

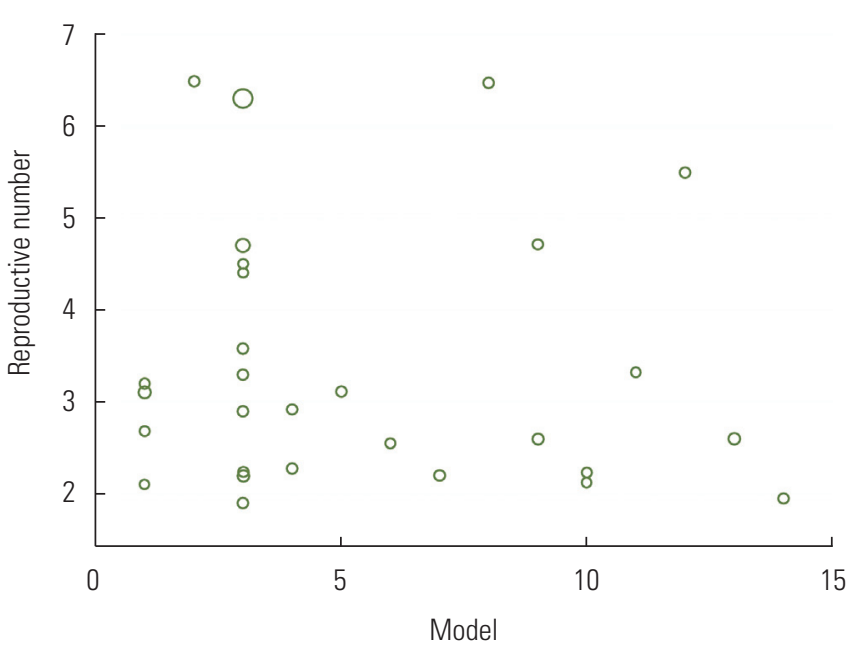

Figure 3. Distribution of the estimated basic reproduction number according to the model used. 
tial growth and a birth-death skyline model; 13, coalescentbased exponential growth and a birth-death skyline model; and 14, type of model not mentioned.

\section{DISCUSSION}

It is necessary to estimate the $R_{0}$ of COVID-19 to determine the severity and size of the pandemic, as well as to design appropriate interventions and responses to protect the population and to control the spread of the disease [35]. The estimated $R_{0}$ value is important in infectious disease epidemiology because the intensity of transmission must be reduced by 1-1/ $R_{0}$ to eliminate the outbreak. For example, at $R_{0}=2.5$, this fraction is $60.0 \%$, but at $R_{0}=3.2$, this fraction is $68.7 \%$. Mathematical models play an important role in decision-making during outbreak control [36]. Our systematic review and meta-analysis found that the overall $R_{0}$ was $3.32(95 \% \mathrm{Cl}, 2.81$ to 3.82$)$, which is higher than the WHO estimates of 1.4 to 2.5 (11) but similar to the results of an earlier review of 12 articles that were conducted in China (11). Our estimation is similar to the $R_{0}$ values estimated for the severe acute respiratory syndrome epidemic in Beijing, China ( $\left.R_{0}=4.91\right)$ [37], and for Middle East respiratory syndrome in Jeddah, Saudi Arabia ( $R_{0}=3.5$ to 6.7) [38]. Such a high $R_{0}$ indicates that the virus can go through at least 3 to 4 generations of transmission [22]. Similar to reviews of $R_{0}$ for other pathogens [39-41], it is important to highlight regarding our results that $R_{0}$ is not an intrinsic characteristic of a given pathogen, but rather describes the transmissibility of that pathogen within a specific population and setting. The estimated $R_{0}$ depends on factors such as social and demographic variables, the estimation method used, the validity of the underlying assumptions, and the biology of the infectious agent. For example, the frequency of contacts may depend on population size and cultural factors, which can vary across regions. In addition, estimates of $R_{0}$ may be somewhat error-prone for reasons such as data insufficiency and the short time period analyzed. As more studies are done and more data are produced, the hope is that this error will be reduced. Our results showed significant heterogeneity among studies $\left(l^{2}=99.3 \%, p\right.$ from the chi-square test for heterogeneity $<0.001$, and $\mathrm{T}^{2}=1.72$ ). One reason for this issue is that it is difficult to calculate the exact number of infected cases during an outbreak. The variation in $R_{0}$ values reported by different studies indicates that precisely estimating $R_{0}$ is rather difficult. Additionally, $R_{0}$ can be affected by environmental factors and modeling methodology [12]. There are many calculation methods for $R_{0}$ [42]. Our review was restricted to Chinese articles. For other countries, surveillance data are needed either to calculate $R_{0}$ or to extrapolate $R_{0}$ estimates from a comparable setting.

It also seems necessary to consider the reasons why high $R_{0}$ values were reported in some studies. Modeling assumptions may be a reason for this issue. Usually, high $R_{0}$ values are calculated in the early stages of an epidemic, both because of the small sample size and the lack of awareness about the disease, which results in inadequate preventive measures being taken. Since the number and patterns of people's contacts in different populations vary because of factors including culture and the level of literacy in the community, $R_{0}$ values vary among different populations and even among subgroups of a single population. In fact, the total value of $R_{0}$ in a population is the average of the $R_{0}$ subtypes in that community. It is therefore important to note that even if the total $R_{0}$ value in a population is low (even less than 1), the likelihood of transmission in some subgroups of that population may still be high. Given the rapid spread of the disease and the dependency of the effectiveness of control measures on factors such as the frequency of asymptomatic infections and the potential for disease transmission before symptom onset, COVID-19 seems to be relatively difficult to control. As a measure used to quantify the transmissibility of a disease in a population, $R_{0}$ is dependent on the population as well as the method of calculation. Our findings suggest that measures such as preventing large gatherings, restricting transportation, and closing schools and universities may be necessary to control this pandemic.

\section{CONCLUSIONS}

Considering the estimated $R_{0}$ for COVID-19, reducing the number of contacts within the population is a necessary step to control the epidemic. So Implementation of the social distancing program, preventing large gatherings, restricting transportation, and closing schools and universities may be necessary to control this pandemic. The estimated overall $R_{0}$ was higher than the WHO estimate.

\section{CONFLICT OF INTEREST}

The authors have no conflicts of interest associated with the material presented in this paper. 


\section{FUNDING}

None.

\section{ACKNOWLEDGEMENTS}

We thank all the authors involved in collecting and processing data.

\section{AUTHOR CONTRIBUTIONS}

Conceptualization:YA, MS. Data curation: YA, MS, MT. Formal analysis: YA, MS. Funding acquisition: None. Methodology: YA, MS. Project administration: MS. Writing - original draft:YA, MS, MT. Writing - review \& editing: YA, MS, MT.

\section{ORCID}

Yousef Alimohamadi https://orcid.org/0000-0002-44809827

Maryam Taghdir https://orcid.org/0000-0003-2853-0196

Mojtaba Sepandi https://orcid.org/0000-0001-6441-5887

\section{REFERENCES}

1. Huang C, Wang Y, Li X, Ren L, Zhao J, Hu Y, et al. Clinical features of patients infected with 2019 novel coronavirus in Wuhan, China. Lancet 2020;395(10223):497-506.

2. Pan F, Ye T, Sun P, Gui S, Liang B, Li L, et al. Time course of lung changes on chest CT during recovery from 2019 novel coronavirus (COVID-19) pneumonia. Radiology 2020;295(3):715721.

3. Boldog P, Tekeli T, Vizi Z, Dénes A, Bartha FA, Röst G. Risk assessment of novel coronavirus COVID-19 outbreaks outside China. J Clin Med 2020;9(2):571.

4. Chen N, Zhou M, Dong X, Qu J, Gong F, Han Y, et al. Epidemiological and clinical characteristics of 99 cases of 2019 novel coronavirus pneumonia in Wuhan, China: a descriptive study. Lancet 2020;395(10223):507-513.

5. Chan JF, Yuan S, Kok KH, To KK, Chu H, Yang J, et al. A familial cluster of pneumonia associated with the 2019 novel coronavirus indicating person-to-person transmission: a study of a family cluster. Lancet 2020;395(10223):514-523.

6. Chen H, Guo J, Wang C, Luo F, Yu X, Zhang W, et al. Clinical characteristics and intrauterine vertical transmission potential of
COVID-19 infection in nine pregnant women: a retrospective review of medical records. Lancet 2020;395(10226):809-815.

7. Lu R, Zhao X, Li J, Niu P, Yang B, Wu H, et al. Genomic characterisation and epidemiology of 2019 novel coronavirus: implications for virus origins and receptor binding. Lancet 2020; 395(10224):565-574.

8. Xu Z, Shi L, Wang Y, Zhang J, Huang L, Zhang C, et al. Pathological findings of COVID-19 associated with acute respiratory distress syndrome. Lancet Respir Med 2020;8(4):420-422.

9. Jung SM, Akhmetzhanov AR, Hayashi K, Linton NM, Yang Y, Yuan $B$, et al. Real-time estimation of the risk of death from novel coronavirus (COVID-19) infection: inference using exported cases. J Clin Med 2020;9(2):523.

10. Wang C, Horby PW, Hayden FG, Gao GF. A novel coronavirus outbreak of global health concern. Lancet 2020;395(10223): 470-473.

11. Liu Y, Gayle AA, Wilder-Smith A, Rocklöv J. The reproductive number of COVID-19 is higher compared to SARS coronavirus. J Travel Med 2020;27(2):taaa021.

12. Delamater PL, Street EJ, Leslie TF, Yang YT, Jacobsen KH. Complexity of the basic reproduction number (R0). Emerg Infect Dis 2019;25(1):1-4.

13. Ghanei Gheshlagh R, Aslani M, Shabani F, Dalvand S, Parizad N. Prevalence of needlestick and sharps injuries in the healthcare workers of Iranian hospitals: an updated meta-analysis. Environ Health Prev Med 2018;23(1):44.

14. Wu JT, Leung K, Leung GM. Nowcasting and forecasting the potential domestic and international spread of the 2019-nCoV outbreak originating in Wuhan, China: a modelling study. Lancet 2020;395(10225):689-697.

15. Shen $M$, Peng Z, Xiao Y, Zhang L. Modelling the epidemic trend of the 2019 novel coronavirus outbreak in China. bioRxiv 2020. doi: https://doi.org/10.1101/2020.01.23.916726.

16. Liu T, Hu J, Kang M, Lin L, Zhong H, Xiao J, et al. Transmission dynamics of 2019 novel coronavirus (2019-nCoV). bioRxiv 2020. doi: https://doi.org/10.1101/2020.01.25.919787.

17. Read JM, Bridgen JR, Cummings DA, Ho A, Jewell CP. Novel coronavirus 2019-nCoV: early estimation of epidemiological parameters and epidemic predictions. MedRxiv 2020. doi: https://doi.org/10.1101/2020.01.23.20018549.

18. Majumder M, Mandl KD. Early transmissibility assessment of a novel coronavirus in Wuhan, China. SSRN 2020. doi: http://dx. doi.org/10.2139/ssrn.3524675.

19. Zhao S, Lin Q, Ran J, Musa SS, Yang G, Wang W, et al. Preliminary estimation of the basic reproduction number of novel 
coronavirus (2019-nCoV) in China, from 2019 to 2020: a datadriven analysis in the early phase of the outbreak. Int J Infect Dis 2020;92:214-217.

20. Imai N, Cori A, Dorigatti I, Baguelin M, Donnelly CA, Riley S, et al. Report 3: transmissibility of 2019-nCoV; 2020 Jan 25 [cited 2020 Apr 20]. Available from: https://www.imperial.ac.uk/mrcglobal-infectious-disease-analysis/covid-19/report-3-transmissibility-of-covid-19/.

21. Riou J, Althaus CL. Pattern of early human-to-human transmission of Wuhan 2019 novel coronavirus (2019-nCoV), December 2019 to January 2020. Euro Surveill 2020;25(4):2000058.

22. Tang B, Wang X, Li Q, Bragazzi NL, Tang S, Xiao Y, et al. Estimation of the transmission risk of the 2019-nCoV and its implication for public health interventions. J Clin Med 2020;9(2):462.

23. Li Q, Guan X, Wu P, Wang X, Zhou L, Tong Y, et al. Early transmission dynamics in Wuhan, China, of novel coronavirus-infected pneumonia. N Engl J Med 2020;382(13):1199-1207.

24. Zhang S, Diao M, Yu W, Pei L, Lin Z, Chen D. Estimation of the reproductive number of novel coronavirus (COVID-19) and the probable outbreak size on the Diamond Princess cruise ship: a data-driven analysis. Int J Infect Dis 2020;93:201-204.

25. Du Z, Wang L, Cauchemez S, Xu X, Wang X, Cowling BJ, et al. Risk for transportation of coronavirus disease from Wuhan to other cities in China. Emerg Infect Dis 2020;26(5):1049-1052.

26. Muniz-Rodriguez K, Chowell G, Cheung CH, Jia D, Lai PY, Lee Y, et al. Epidemic doubling time of the COVID-19 epidemic by Chinese province. medRxiv 2020. doi: https://doi.org/10.1101/ 2020.02.05.20020750.

27. Zhou C. Evaluating new evidence in the early dynamics of the novel coronavirus COVID-19 outbreak in Wuhan, China with real time domestic traffic and potential asymptomatic transmissions. medRxiv 2020. doi: https://doi.org/10.1101/2020. 02.15.2002344.

28. Liu T, Hu J, Xiao J, He G, Kang M, Rong Z, et al. Time-varying transmission dynamics of novel coronavirus pneumonia in China. bioRxiv 2020. doi: https://doi.org/10.1101/2020.01.25. 919787.

29. Li R, Pei S, Chen B, Song Y, Zhang T, Yang W, et al. Substantial undocumented infection facilitates the rapid dissemination of novel coronavirus (SARS-CoV2). Science 2020;368(6490): 489-493.

30. Park SW, Bolker BM, Champredon D, Earn DJ, Li M, Weitz JS, et al. Reconciling early-outbreak estimates of the basic reproductive number and its uncertainty: framework and applications to the novel coronavirus (SARS-CoV-2) outbreak. medRxiv
2020. doi: https://doi.org/10.1101/2020.01.30.20019877.

31. Shao N, Cheng J, Chen W. The reproductive number R0 of COVID-19 based on estimate of a statistical time delay dynamical system. medRxiv 2020. doi: https://doi.org/10.1101/2020.02.1 7.20023747.

32. Zhang KK, Xie L, Lawless L, Zhou H, Gao G, Xue C. Characterizing the transmission and identifying the control strategy for COVID-19 through epidemiological modeling. medRxiv 2020. doi: https://doi.org/10.1101/2020.02.24.20026773.

33. Lai A, Bergna A, Acciarri C, Galli M, Zehender G. Early phylogenetic estimate of the effective reproduction number of SARSCoV-2. J Med Virol 2020;92(6):675-679.

34. Sanche S, Lin YT, Xu C, Romero-Severson E, Hengartner NW, $\mathrm{Ke} \mathrm{R}$. The novel coronavirus, 2019-nCoV, is highly contagious and more infectious than initially estimated. arXiv 2002:03268.

35. Kwok KO, Tang A, Wei VW, Park WH, Yeoh EK, Riley S. Epidemic models of contact tracing: systematic review of transmission studies of severe acute respiratory syndrome and Middle East respiratory syndrome. Comput Struct Biotechnol J 2019;17: 186-194.

36. Egger M, Johnson L, Althaus C, Schöni A, Salanti G, Low N, et al. Developing WHO guidelines: time to formally include evidence from mathematical modelling studies. F1000Res 2017; 6:1584.

37. Gumel AB, Ruan S, Day T, Watmough J, Brauer F, van den Driessche $P$, et al. Modelling strategies for controlling SARS outbreaks. Proc Biol Sci 2004;271(1554):2223-2232.

38. Majumder MS, Rivers C, Lofgren E, Fisman D. Estimation of MERS-coronavirus reproductive number and case fatality rate for the Spring 2014 Saudi Arabia outbreak: insights from publicly available data. PLoS Curr 2014;6:ecurrents.outbreaks.98d 2f8f3382d84f390736cd5f5fe133c.

39. Ridenhour B, Kowalik JM, Shay DK. Unraveling R0: considerations for public health applications. Am J Public Health 2014; 104(2):e32-e41.

40. Biggerstaff M, Cauchemez S, Reed C, Gambhir M, Finelli L. Estimates of the reproduction number for seasonal, pandemic, and zoonotic influenza: a systematic review of the literature. BMC Infect Dis 2014;14:480.

41. Johansson MA, Hombach J, Cummings DA. Models of the impact of dengue vaccines: a review of current research and potential approaches. Vaccine 2011;29(35):5860-5868.

42. Breban R, Vardavas R, Blower S. Theory versus data: how to calculate R0? PLoS One 2007;2(3):e282. 